\title{
A Speech Controlled Artificial Limb Based on DSP Chip
}

\author{
Chih-Lung Lin*, Shao-Cheng Wang, Hen-Chong Wu, Shuenn-Tsong Young, \\ Maw-Huei Lee, and Te-Son Kuo \\ Department of Electrical Engineering National Taiwan University Taipei 10617,Taiwan \\ *E-mail: cllin@cc.ee.ntu.edu.tw
}

\begin{abstract}
An speech signal pattern recognition DSP-based system is constructed for real-time control of artificial limb through precise identification of speech and speech command. The portable DSP-based system provides an effective method to provide multiple control commands than the electomyographic signals from the amputation stump or residual limb, the traditional prosthetic arm can not archive many complex actions because the EMG of residual limb is hard to detach many different control signals. Goals of multiple command recognition and low cost were archived by using a portable DSP-based system.
\end{abstract}

\section{INTRODUCTION}

Today's technology is apparently increasing in the classification of speech pattern and the operation speech of microprocessor[1]. Many applications are used in biomedical engineering[2]. In fact, the speech control is useful to help the amputee to control the electrical powered artificial limb. These functions, such as humeral rotation in, humeral rotation out, elbow flexion, elbow extension, forearm rotation, wrist pronation, wrist supination, and the hand manipulation action including the coordination among the fingers, thumb, and the palm to grasp, and abandon are provided by the upper limb of normal man. However, the electrical powered artificial limb controlled by the EMG signals developed in the muscles left in the residual limbs can not do such as much work because it is difficult for the residual muscle to provide these complex many different actions. The mechanic architecture of artificial limb can be provide complex action such as the normal. The famous Utah artificial limb provided many action functions[3]. However, it is restricted to the sufficient commands from the residual muscle. It is not convenient to use for the amputee. Traditionally, the artificial limb can accept no more than ten commands from the residual muscle to control the different actions.

Now, we propose a portable DSP-based system for speech recognition to provide enough commands to correctly control the electrical powered artificial limb. The system can help the amputees recover the function of limb and hand to do more than it was used in the control of EMG at his will, and it is convenient and low cost for the amputees.

\section{MATERIALS AND METHODS}

\section{A. System Description}

The system, shown in Fig. 1, contains a microphone, analog interface circuit (AIC), 50-MFLOPS 32 bits floating DSP, $128 \mathrm{k} * 8$ flash ROM and $128 \mathrm{k} * 32$ high speed SRAM (20ns), output channel of the AIC and the artificial limb.

Through a directional microphone which collects the sound of voices only within an angle of $30^{\circ}$ from the source, human voice is gathered into the system. The analog interface circuit (AIC) consists of an $A / D$ converter and a bandpass filter. Human voice is thus converted into digital signal and enters the Digital Signal Processor (DSP), TMS320C31. Here, $10 \mathrm{KHz}$ speech data sampling rate is adapted. This DSP is responsible for speech recognition. After the recognition, the system output the relative command to the DAC to control the powered electric artificial limb of the amputee to do the fine action at his will.

\section{B. Software Skeleton}

Fig 2. Shows the flow chart of speech signal processing. The first three blocks of this flow are the pre-processes of the time domain waveform. With these stages the input speech signals will be clearer and more meaningful to the later mathematical models, and a faster processing rate is also achieved. Auto-correlation and LPC coefficients are calculated and further processed to get the Cepstrum coefficients with Durbin numerical method. Throughout these stages, we define every $120 \mathrm{~ms}$ as a frame and each frame overlaps the prior frame's last $60 \mathrm{~ms}$. On the other hand, we extract 10-order cepstrum coefficients for each frame. Operation of the recognition engine is based on "frame by frame" comparison. Since the amount of the frame to be compared and the frame stored in the database are different . That is because the voice length of a word spoken by a person, but at different time is quite different. This is illustrated in Fig. 3. To solve the problem, The DTW (Dynamic Time Warping) algorithm is used. This algorithm allows the speaking speed ranging from a fraction of 0.5 to a multiple of 2 . In this range, a dynamic disposal will achieve. For Example, the frame to be compared is N[3] , the 
allowable frame stored in database will be $\mathrm{M}[2], \mathrm{M}[3]$, $M[4]$. i.e.

$$
D(m, n)=\min \left(\begin{array}{l}
D(m-1, n) \\
D(m-1, n-1) \\
D(m-1 . n-1)
\end{array}\right)
$$

$\mathrm{m}:$ the frame to be compared

$\mathrm{n}$ : the frame stored in database

With proper training steps, the speech commands' characteristic coefficients database would be stored in the memory section, later when recognition mode is operated, the recognition engine would compare each speech input with the predefined database to identify certain commands . Once a command is identified, C31 would utilize its serial port to drive the AIC output channel as controlling the electrical artificial limb to do certain actions.. The compared result will then be further processed to decide which action will be performed by the artificial limb, or just to be discarded

\section{RESULTS}

A real-time electrical artificial limb with a humanmachine interface based on a speech recognition system is built. Moreover, it takes only $0.3 \mathrm{sec}$ per recognition. Realtime system performance is achieved. In the system, 5 predefined speech commands, such as hand "grab", "release", "stop", "slow speed", and "quick speed" are used to control the artificial arm. Their recognition rate can reach up $95 \%$.

the artificial limb can be controlled easily and correctly. The command of the system can be extend to many instructions to control tendon-operated, multiple-degree-offreedom robotics hand with the speech recognition in the future.

\section{REFERENCES}

[1] C. S. Myers, and L. R. Rabiner, "Connected digitrecognition using a level-building DTW algorithm," IEEE Trans. Acoust., Speech, Signal Processing, vol. 29, pp. 351-363, Jun. 1981.

[2] P. Nisbet, "Integrating assistive technologies: current practices and future possibilities," Med. Eng. Phys. vol. 18, pp. 193-202, 1996.

[3] S. C. Jacobsen, J. E. Wood, D. F. Knutti, and K. B. Biggers, "The UTAH/M.I.T Dextrous Hand: Work in Progress," The international Journal of Robotics Research, pp. 21-50, 1984.

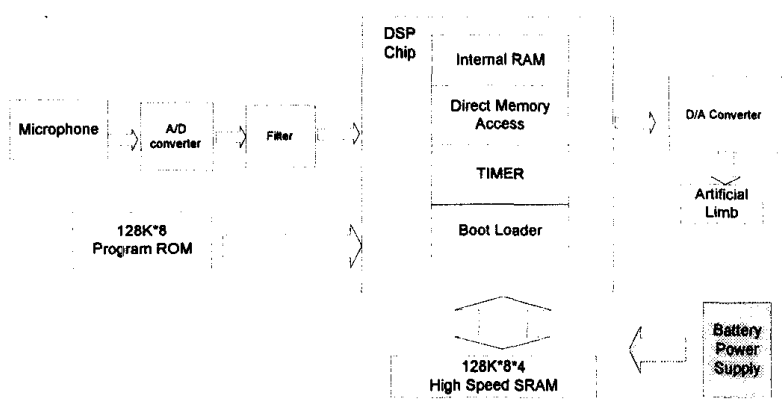

Fig 1. System block diagram

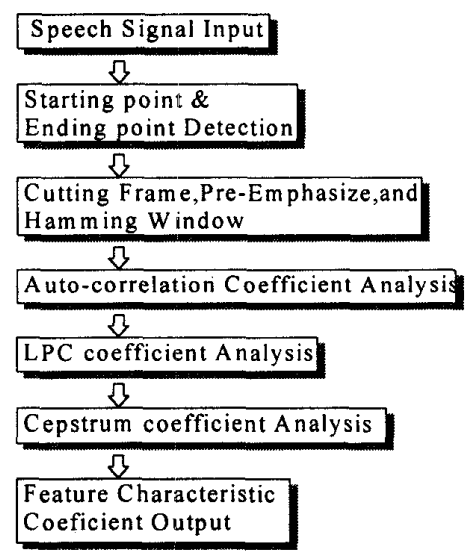

Fig. 2. Speech Signal Processing

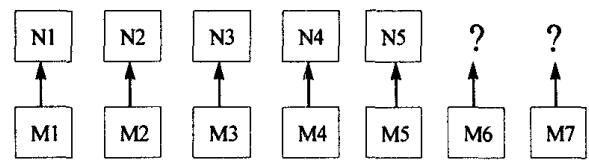

Fig 3. Example encountered in frame-by-frame camparison 